\title{
Microwave Nondestructive Evaluation of Dielectric Materials with a Metamaterial Lens
}

\author{
D. Shreiber ${ }^{*}$, M. Gupta ${ }^{*}$ and R. Cravey ${ }^{* *}$ \\ *Department of Electrical and Computer Engineering, University of Virginia, \\ Charlottesville, VA 22904 \\ ${ }^{* *}$ Electromagnetics and Sensors Branch, NASA Langley Research Center,
} Hampton, VA 23681

\begin{abstract}
A novel microwave Nondestructive Evaluation (NDE) sensor was developed in an attempt to increase the sensitivity of the microwave NDE method for detection of defects small relative to a wavelength. The sensor was designed on the basis of a negative index material (NIM) lens. Characterization of the lens was performed to determine its resonant frequency, index of refraction, focus spot size, and optimal focusing length (for proper sample location). A sub-wavelength spot size $(3 \mathrm{~dB})$ of $0.48 \lambda$ was obtained. The proof of concept for the sensor was achieved when a fiberglass sample with a $3 \mathrm{~mm}$ diameter through hole (perpendicular to the propagation direction of the wave) was tested. The hole was successfully detected with an $8.2 \mathrm{~cm}$ wavelength electromagnetic wave. This method is able to detect a defect that is $0.037 \lambda$. This method has certain advantages over other far field and near field microwave NDE methods currently in use.
\end{abstract}

Introduction. There are a wide variety of nondestructive evaluation (NDE) methods presently in use by industry and under development, including ultrasonic, laser, visual, thermography, microwave and other methods. Each method has its own advantages and disadvantages. For example, thermography can cause damage to a sample due to temperature excursions from a flash pulse. Laser methods frequently require a 
thermal/mechanical load on the sample. Ultrasonic methods require extensive data interpretation, and also require a coupling material.

In addition to relatively high spatial resolution compared to some NDE techniques, microwave NDE techniques also have other advantages. Microwave NDE techniques have the potential to penetrate deeper into materials such as composites and other dielectrics as compared to ultrasonic techniques ${ }^{1}$. Also, microwave NDE is relatively inexpensive, fast ${ }^{2}$ and does not require extensive data analysis.

Microwave NDE sensors typically operate in two modes - far field and near field. The near field method is generally used at standoff distances below $10 \mathrm{~mm}$, whereas the far field method is used at greater standoff distances. Although far field information is easier to obtain, there are several disadvantages of using it. For example, many critical areas that require testing are situated at the edges, corners or joints of the sample and unwanted reflections from these structures could limit the utility of this method ${ }^{3}$. The spatial resolution of the near field mode is generally better than that of the far field mode. The disadvantage of the near field mode is that the standoff distance has to be maintained relatively close to the sample, making it more difficult to implement. Although using the optimum standoff distance can enhance measurement sensitivity of the near field mode, small deviations from this standoff can significantly degrade this sensitivity ${ }^{3}$. Determination of an optimal standoff distance for the near field case is an additional complication for the user of the system.

Far field mode results for spatial resolution are independent of the standoff distance, and thus this method is less sensitive to sensor positioning. Spatial resolution of the far field is approximately equal to the wavelength. The spatial resolution in the near field mode is 
primarily a function of the probe dimensions and not necessarily of the wavelength. The purpose of this work is to develop a new microwave NDE sensor which will combine the advantages of both the near field and the far field modes of NDE. This sensor would operate at standoff distances typical for the far field mode, the probe size and the resolution will be typical for the near field mode and the dependence of the resolution on standoff distance could be easily established. Since the probe size will be smaller than the wavelength, a longer wavelength could be used for deep penetration into a dielectric material without compromising the resolution, and the edge effects could be avoided. This paper describes a concept for a microwave NDE system which achieves the desirable characteristics mentioned above by including a negative index material (NIM) lens. The following sections give an overview of NIMs, a description of the prototype microwave NDE system that has been developed and tested, experimental results, and conclusions.

Negative index materials. Peculiar properties such as negative index of refraction, reversed Doppler effect and other effects were predicted for materials with simultaneously negative permittivity and permeability in $1968^{4}$. Such a medium was called "left-handed" because the electric field (E), magnetic field (H) and propagation (k) vectors would form a left handed triplet. This fact allows for construction of a flat lens. It was shown by J. Pendry ${ }^{5}$ that when we have an "ideal" NIM with the index of refraction $n=-1$ exactly, the sum of the distances of the object to the lens interface $\left(d_{1}\right)$ and lens interface to image $\left(\mathrm{d}_{2}\right)$ equals the thickness of the lens $(\mathrm{t})$ as shown in Fig. 1 and Eqn (1): 


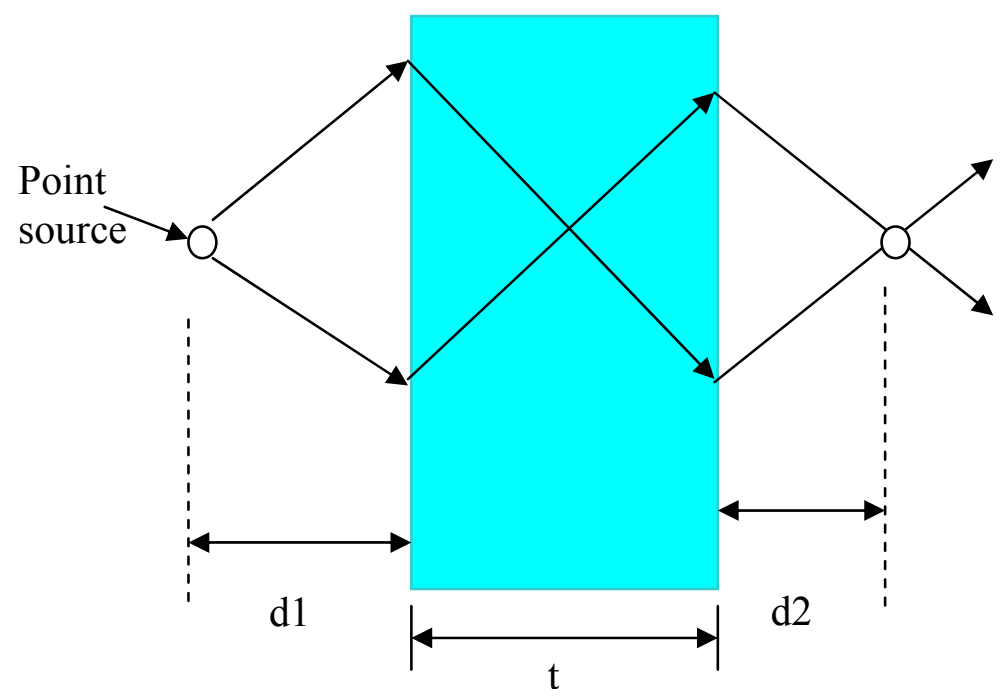

Fig.1 Ray Diagram for a NIM Lens

$\mathrm{d}_{1}+\mathrm{d}_{2}=\mathrm{t}$

It was also shown by Pendry that the diffraction limit for image resolution (approximately one wavelength) would not apply in the case of this "ideal lens" or "perfect lens". In reality it is very hard to obtain a material which has $n=-1$ exactly, but Equation 1 gives a very good estimate of the location of the focused spot even for an "imperfect" NIM lens if the distance from the object to interface and the thickness of the lens are known. The exact distance of the focused spot can be determined experimentally in this case.

Unfortunately, such NIMs do not exist in nature. The electric and magnetic properties of materials are described by dielectric permittivity $(\varepsilon)$ and magnetic permeability $(\mu)$. Together they determine the response of a material to electromagnetic radiation. In order for an electromagnetic wave of a certain frequency to propagate in a material both $\varepsilon$ and $\mu$ have to be positive at that frequency. Veselago ${ }^{4}$ had shown that a material requires both permittivity and permeability to be negative in order to exhibit the effects that were discussed earlier. $\varepsilon$ is known to be negative for some naturally occurring materials at certain frequencies. For example, $\varepsilon$ is negative below the plasma frequency for metals. 
However, for proper operation of the lens, the frequency in use has to be close to the plasma frequency ${ }^{6}$. The plasma frequency for metals is normally in the ultraviolet region. In order to reduce the plasma frequency to the microwave region, it was suggested that an array of thin metallic wires could be used ${ }^{7}$.

There are no materials in nature with negative $\mu$. Materials which are manufactured from periodic structures designed to resonate at a particular frequency can be demonstrated to exhibit negative $\varepsilon$ and $\mu$ simultaneously at that frequency. Such "artificial" materials, or engineered materials, are called metamaterials. In 1999 Pendry et al. ${ }^{8}$ introduced a new structure called a split ring resonator (SRR) which exhibits negative magnetic permeability at certain frequencies (Fig.2). The SRR structure can be easily realized as a metallized pattern on a printed circuit board. The structure obtained when SRRs were combined with an array of thin metallic wires paved the way for the design of a metamaterial that would exhibit the negative index of refraction characteristics that are described above.

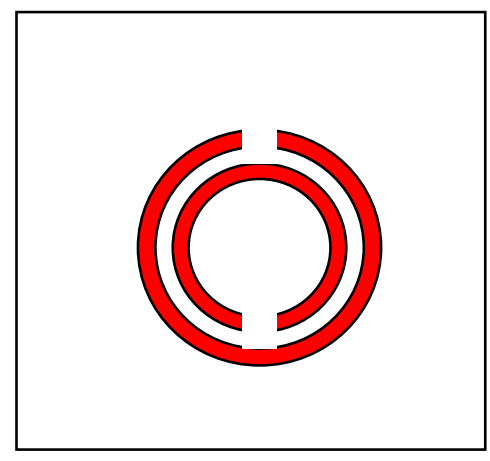

Fig.2 A schematic of a Split Ring Resonator (SRR)

The experimental verification of the negative refractive index in these materials was first performed by R.A. Shelby et al. in $2001^{9}$. Today metamaterials which exhibit left handed 
properties are being investigated extensively. The metamaterial can be engineered in a way that it would exhibit a negative refractive index at frequencies that range from low $\mathrm{RF}$ to visible light. The metamaterial structures are resonant and transmit EM radiation (when the material exhibits negative $\varepsilon$ and $\mu$ ) only at a certain range of frequencies. This kind of metamaterial is known to be a lossy structure which makes it very hard to obtain the perfect lens condition. Some previous experimental work shows that a focus spot size of $0.4 \lambda^{10}$ was achieved-.

Experimental Details. Although the ultimate goal in designing a NIM lens for a NDE sensor is to achieve high spatial resolution (by the use of higher frequency resonances for the NIM), we concentrate in this work on proving the principle of operation for the NIM using a metamaterial that exhibits resonance at relatively low frequency range (around 3$4 \mathrm{GHz}$ ). The reason for this lower frequency range to demonstrate proof of concept is that such a lens is much easier to manufacture than a lens for higher frequency range. Also, a lens for the $3-4 \mathrm{GHz}$ frequency range has previously been designed and tested by Aydin, et. $\mathrm{al}^{10}$, so the design is already known to exhibit focusing and negative index of refraction in the stated frequency range. The dimensions of the lens are $372 \mathrm{~mm} \times 186$ mm x $96 \mathrm{~mm}$ or $40 \times 20 \times 10$ unit cells. Each unit cell measures $9.3 \mathrm{~mm}$ in length in each direction and contains 2 SRRs on the front side and 2 wires on the back side of the printed circuit board in a "wine crate" configuration (see Fig. 3).
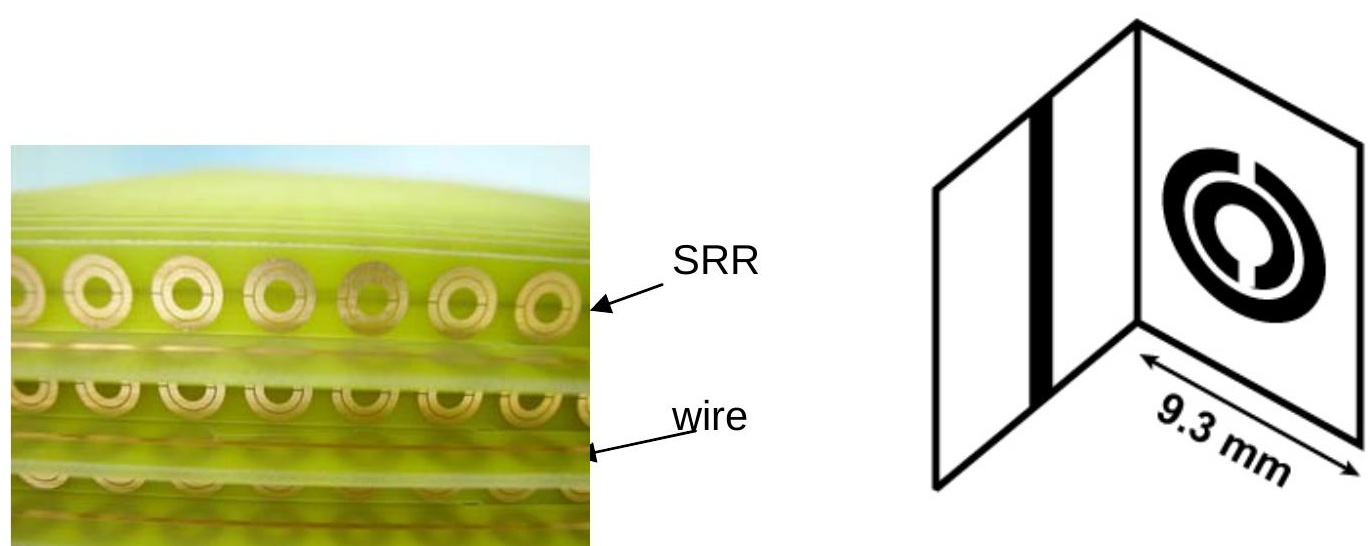
Fig.3 (a) Metamaterial lens tested by the authors for frequencies in the range of 3$4 \mathrm{GHz}$, (b) a sketch of a unit cell for the lens

In order to confirm the resonant frequency of this material a transmission frequency sweep experiment was performed for a relatively wide range of frequencies $(3-7 \mathrm{GHz})$. The following equipment was used for the frequency sweep experiment: a Hewlett Packard (HP) 8510 Network Analyzer, 2 Atlantic Electronics Limited (AEL) horn antennas (broad band $2 \mathrm{GHz}-18 \mathrm{GHz}$ ) and the metamaterial lens which was embedded into a fixture that was covered with absorber in order to maximize the isolation of the two antennas. A sketch of the fabricated NIMs lens system is depicted in Fig. 4 and photographs of the system are provided in Fig.5. 


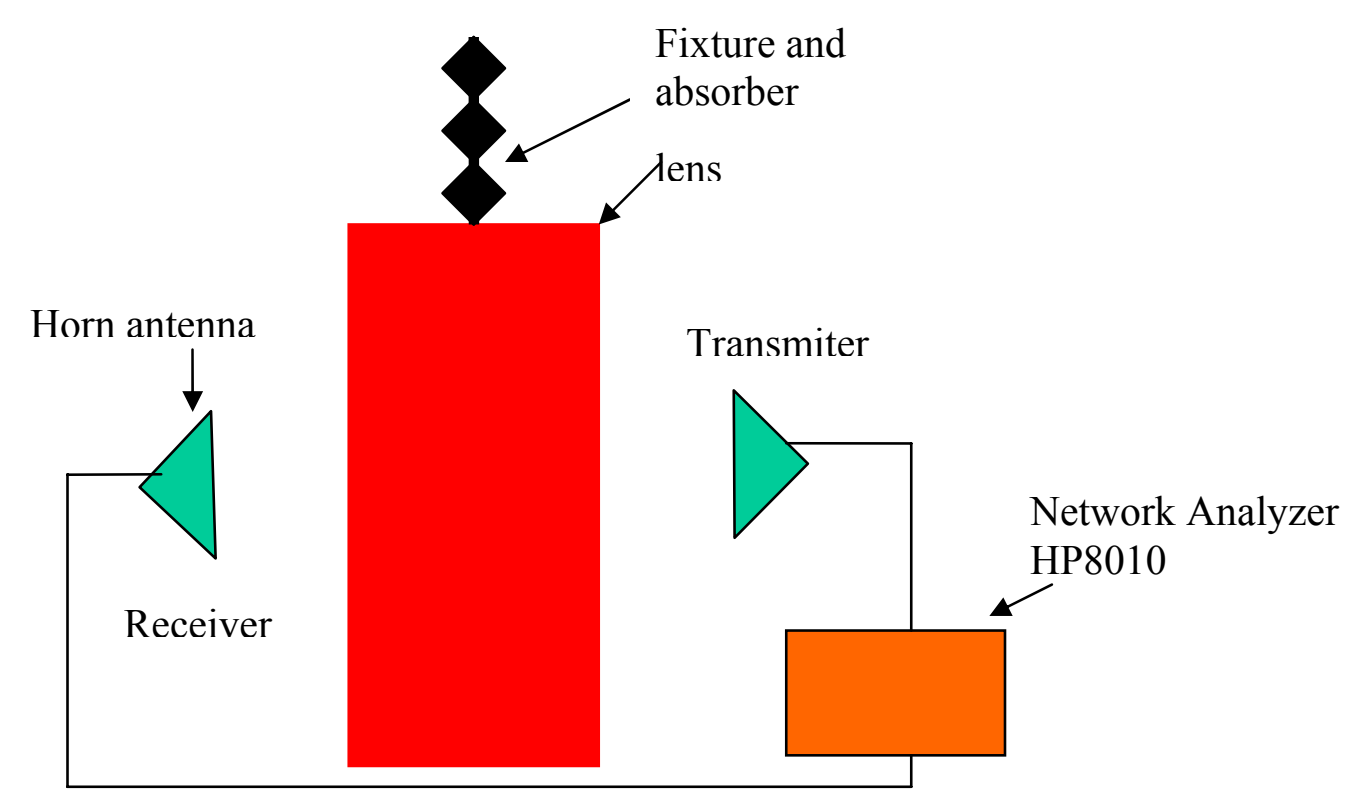

Fig.4 A sketch of the experimental system for the frequency sweep experiment
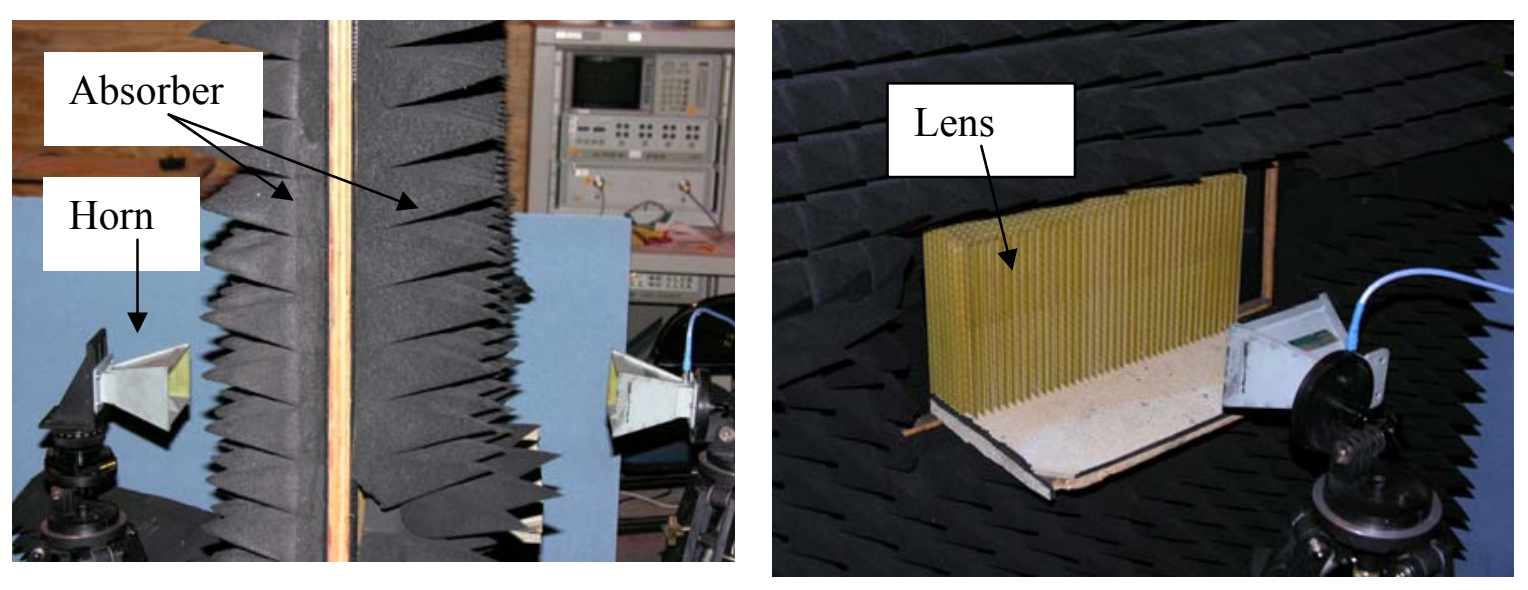

Fig.5 Photographs of the experimental setup

Further characterization of the lens was performed in terms of the refractive index and the focus spot size. Fig. 6 shows the experimental setup used for the measurements.

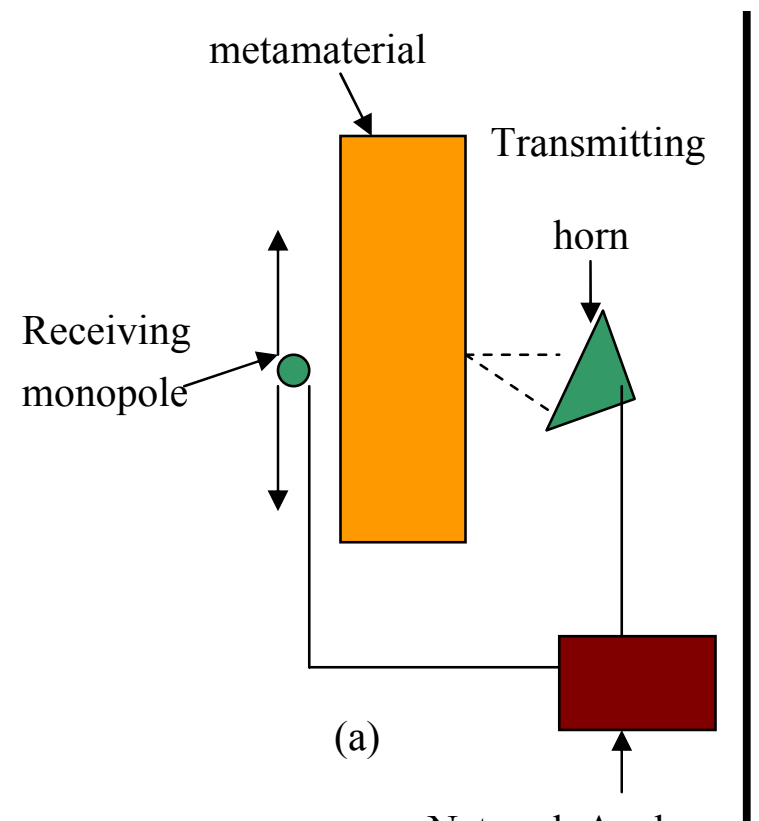

Network Analyzer

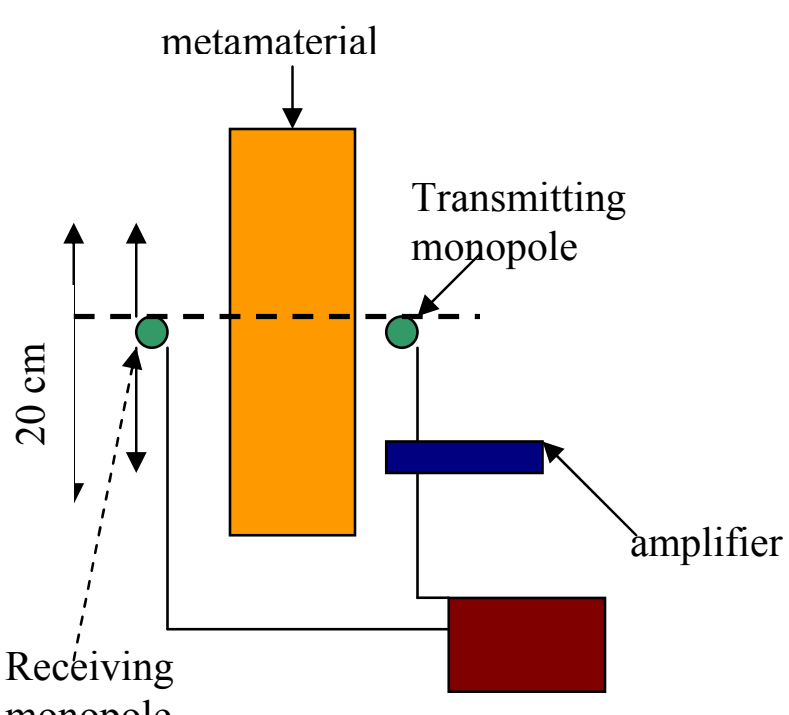

(b) Network Analyzer 


\section{Fig.6 A sketch of two experiments that were designed to determine (a) index of refraction for the metamaterial and (b) the focus spot size}

In the first experiment (Fig. 6(a)), a monopole was used to scan the field on the opposite side of the lens from a transmitting horn antenna for two different incident directions. First the horn was pointed normal to the plane of the lens to establish a baseline point of maximum transmission and then tilted 15 degrees relative to normal incidence. The scanning monopole was brought as close as possible to the NIM lens and the point of highest energy density along the scan was determined and was taken as a point of refraction (see Fig. 6 (a)). The index of refraction can be determined from Snell's law when the distance $(12 \mathrm{~cm})$ from the horn antenna to the metamaterial lens and its thickness $(9.3 \mathrm{~cm})$ are known. This experiment was repeated for several frequencies around the resonant peak in order to establish the frequency where the metamaterial would exhibit an index of refraction closest to $n=-1$.

In order to determine the focus spot of the metamaterial lens, the experiment which is depicted in Fig. 6 (b) was performed for several frequencies. The stationary monopole was positioned arbitrarily at $6 \mathrm{~cm}$ away from the lens surface so that it is within the distance specified by Equation 1. In the ideal case, for $n=-1$, Equation 1 gives the distance $\mathrm{d}_{1}$ for the focused spot as $3.3 \mathrm{~cm}$,. Therefore, the scanning monopole was operated at distances that were closer to the lens than $3.3 \mathrm{~cm}$. Both the frequency and the scanning distance were varied to find the optimal focusing. Since the signal which was 
emitted by the transmitting monopole was weak and the losses in the lens were high, a 20 $\mathrm{dB}$ amplifier was used to enhance the signal before going into the lens.

Prototype NDE sensor. After the lens was characterized and it was established that a focused spot smaller than a wavelength was possible at certain frequencies (3.6-3.7 GHz), the first NDE sensor was designed based on this lens. Fig. 7 depicts a sketch of the sensor.

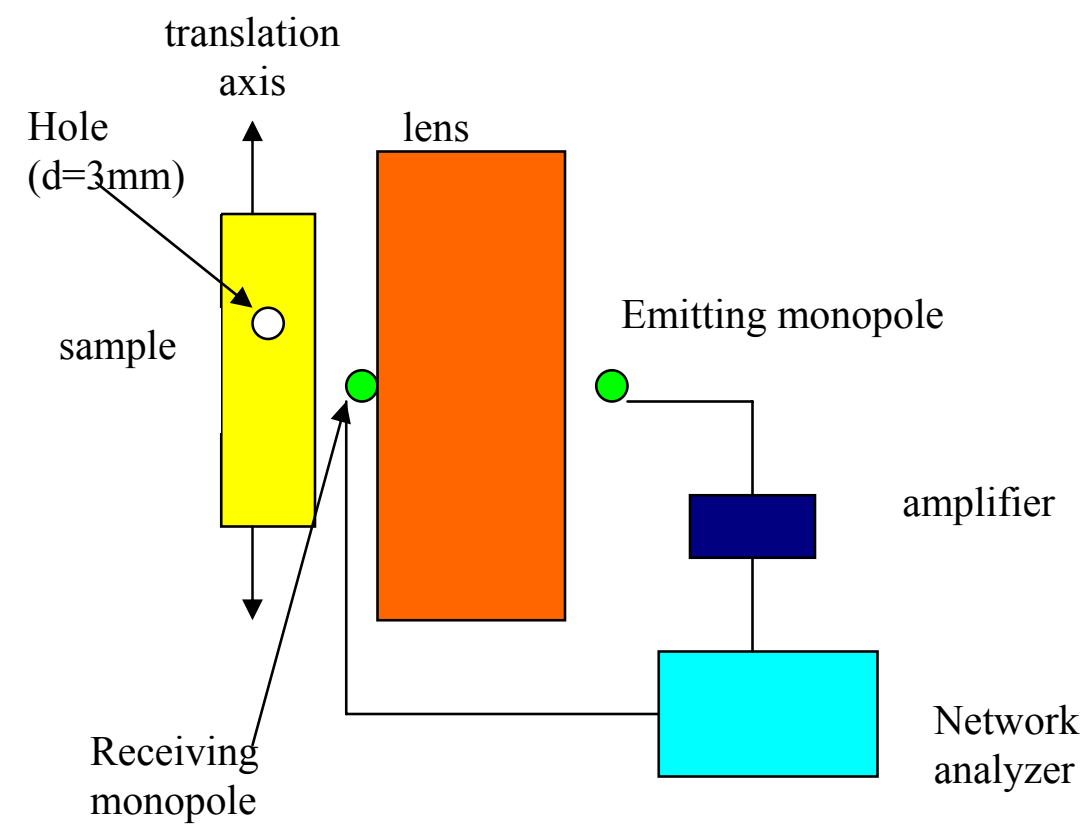

Fig.7 Sketch of the NDE sensor system used in this work

For evaluation of the sensor for NDE applications, a test sample was constructed to investigate discontinuities in a dielectric material. A fiberglass sample $(\varepsilon=4.8)$ was used as a test material. A $3 \mathrm{~mm}(0.037 \lambda)$ diameter hole was drilled perpendicular to the translation axis as shown in Fig.8, and a cylindrical fiberglass filler was also fabricated, in order to compare a sample with and without a hole. 


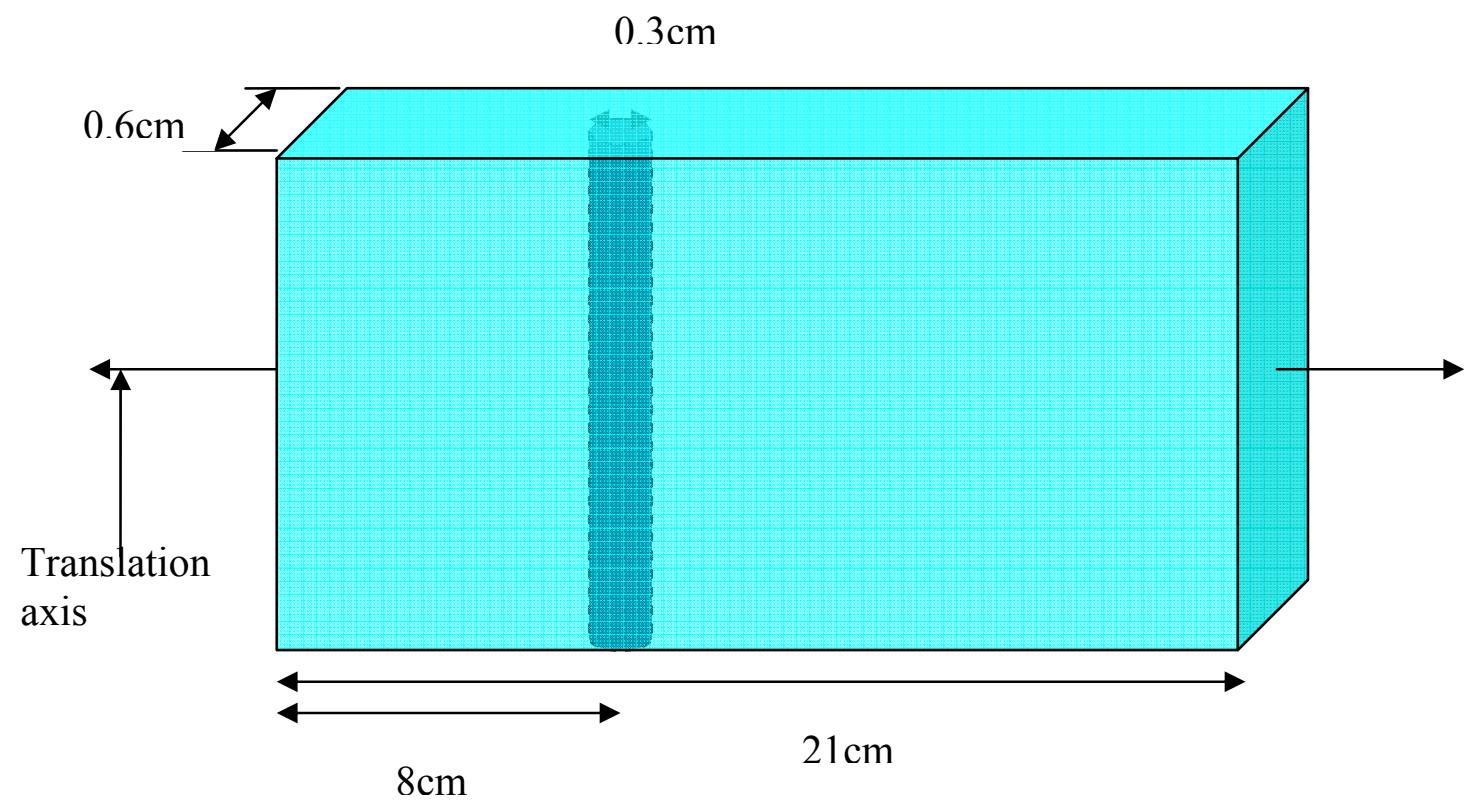

Fig.8 Front view of the sample (fiberglass)

The radiating monopole was located $1.5 \mathrm{~cm}$ away from the lens surface to allow for a larger distance to the focus spot on the other side of the lens to accommodate the physical dimensions of the test sample $\left(\mathrm{d}_{2}\right.$ in eqn 1$)$. The signal emitted from the monopole was amplified in order to enhance the signal to noise ratio. Ideally, the same monopole could be used to detect the reflected signal, but because the metamaterial is lossy (only around $1 \%$ of the incident energy is transmitted) an additional monopole was placed on the other side of the lens to detect the reflected signal from the sample. The sample was positioned at a distance of $5 \mathrm{~cm}$ away from the lens where the focusing occurs. The frequency used in the experiment was $3.65 \mathrm{GHz}$ (the reason for the shift from the $3.66 \mathrm{GHz}$ used in the lens characterization experiments will be explained in the Results and Discussion section). The sample was attached to an XYZ positioner and scanned $20 \mathrm{~cm}$ along the translational axis as shown in Fig.7. Scans were performed both with and without a hole 
by inserting and then removing the fiberglass plug described above. This allowed for background subtraction to be performed, providing a response that should be characteristic of the hole alone.

\section{Results and Discussion.}

Transmission Frequency Sweep. The result that was obtained from the frequency sweep experiment is shown in Fig.9:

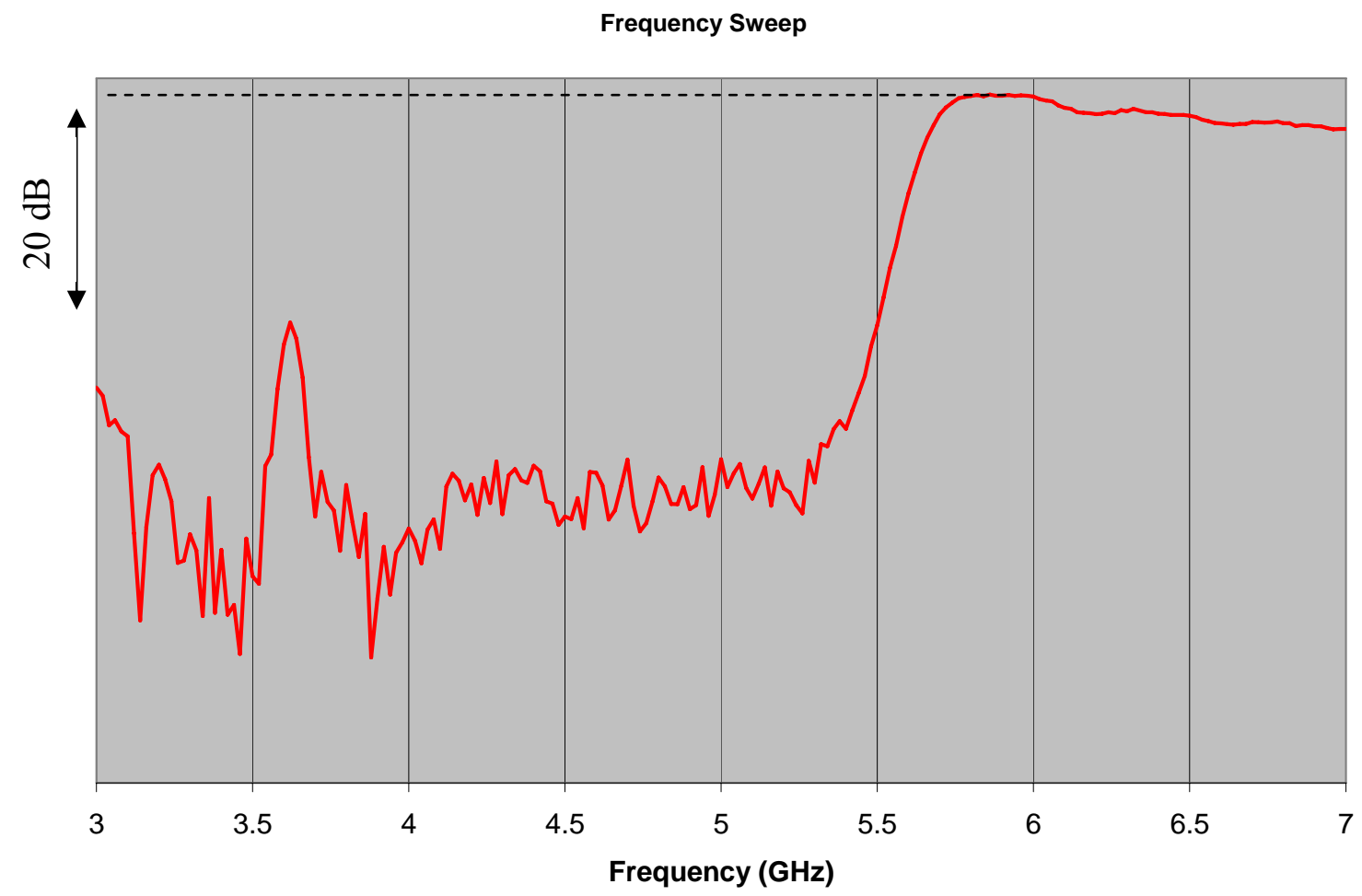

Fig.9. Results of transmission frequency sweep for the metamaterial lens

From the plot shown in Fig.9 it can be determined that the NIM lens has a resonant peak at $\mathrm{f}=3.625 \mathrm{GHz}$. The shape of the transmission curve is similar to the one that was described by K. Aydin et $\mathrm{al}^{10}$. High transmission at frequencies higher than $5.5 \mathrm{GHz}$ was due to simultaneously positive $\varepsilon$ and $\mu$ at these frequencies. 
The NIM lens structure was modeled using Ansoft's HFSS ${ }^{\text {TM }}$ software. A computational model, once validated, will serve as an important design tool to construct lenses which are resonant at specific frequencies (which determines the spatial resolution of the lens). Three unit cells were used along the direction of the wave propagation which was found sufficient ${ }^{11}$ to describe the behavior of a system. Perfect $\mathbf{E}$ boundary conditions were used for $\mathbf{E}$ perpendicular to the $\mathrm{z}$ direction and perfect $\mathbf{H}$ boundary conditions perpendicular to y direction. The incident electric field $\mathbf{E}$ was directed parallel to the wires (in the y direction). Fig.10 shows the image of the HFSS model, and Fig.11 shows the results obtained from the model.

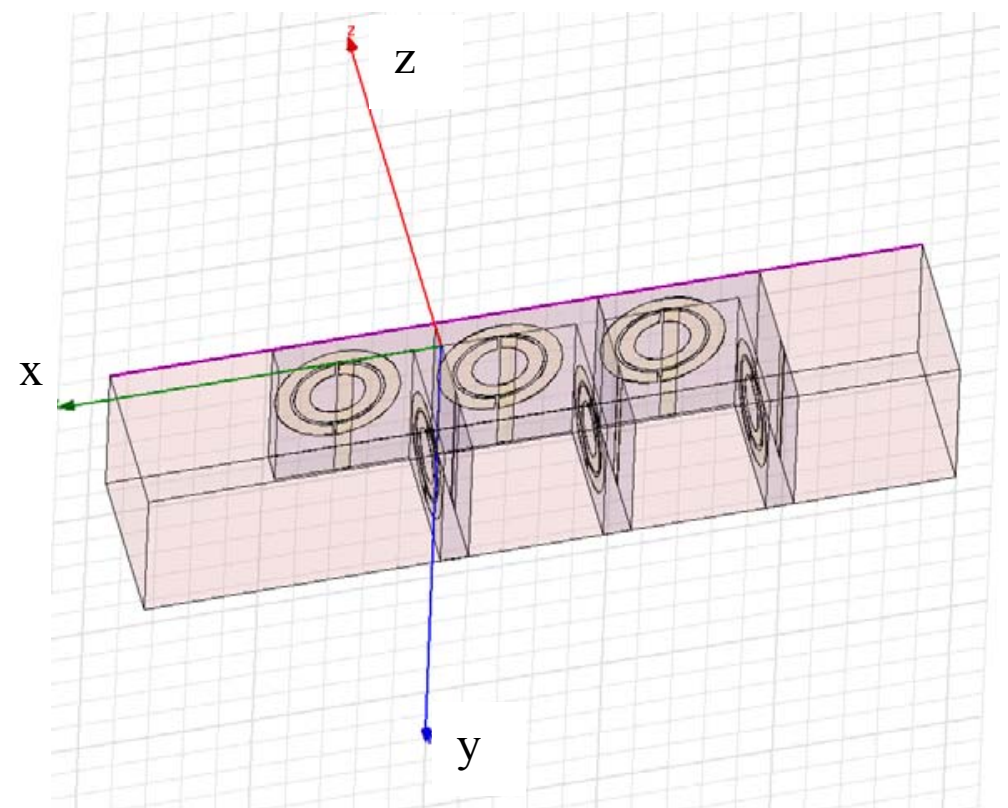

Fig.10 Image of the model 


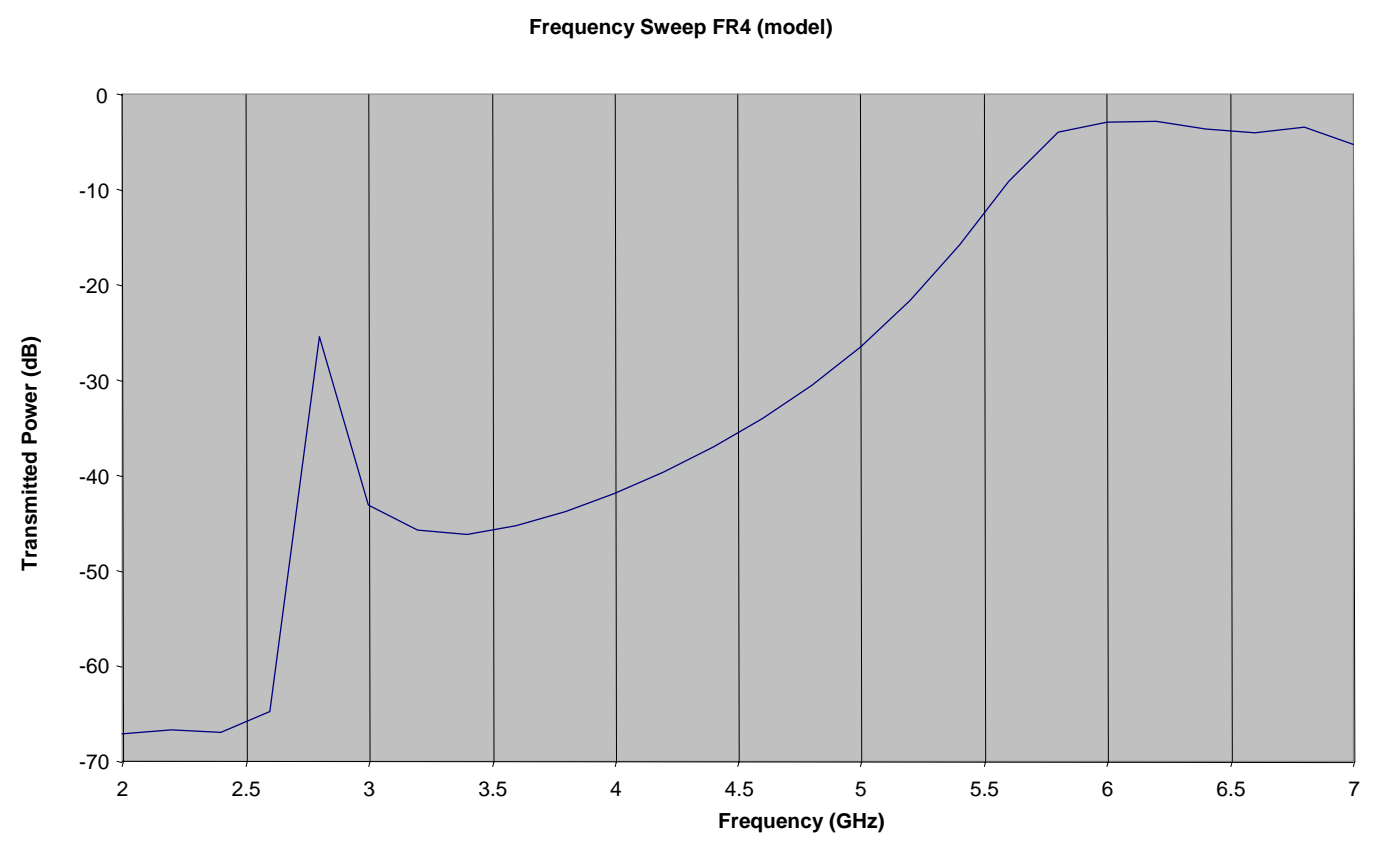

Fig.11 Modeling results for transmitted power as a function of incident microwave

\section{frequency}

Figure 11 shows a transmission plot very similar to the experimental result of the frequency sweep for the NIM lens. The calculated resonant peak was at $2.8 \mathrm{GHz}$. The difference of $0.8 \mathrm{GHz}$ between the experimental and modeled results could be attributed to a difference in the dielectric constants of the substrate material for the lens (FR4) as specified in the HFSS ${ }^{\mathrm{TM}}$ material database, and the actual dielectric constant in the experiment. Some misalignment of the elements of the lens may account for some discrepancy between measured and computed results.

Index of Refraction Determination. Fig.12 shows the results of a normally incident scan and one performed at $15^{\circ}$. Fig. 13 shows the results on refractive index variation with incident microwave signal frequency. 
3.64 GHz 0->15 deg

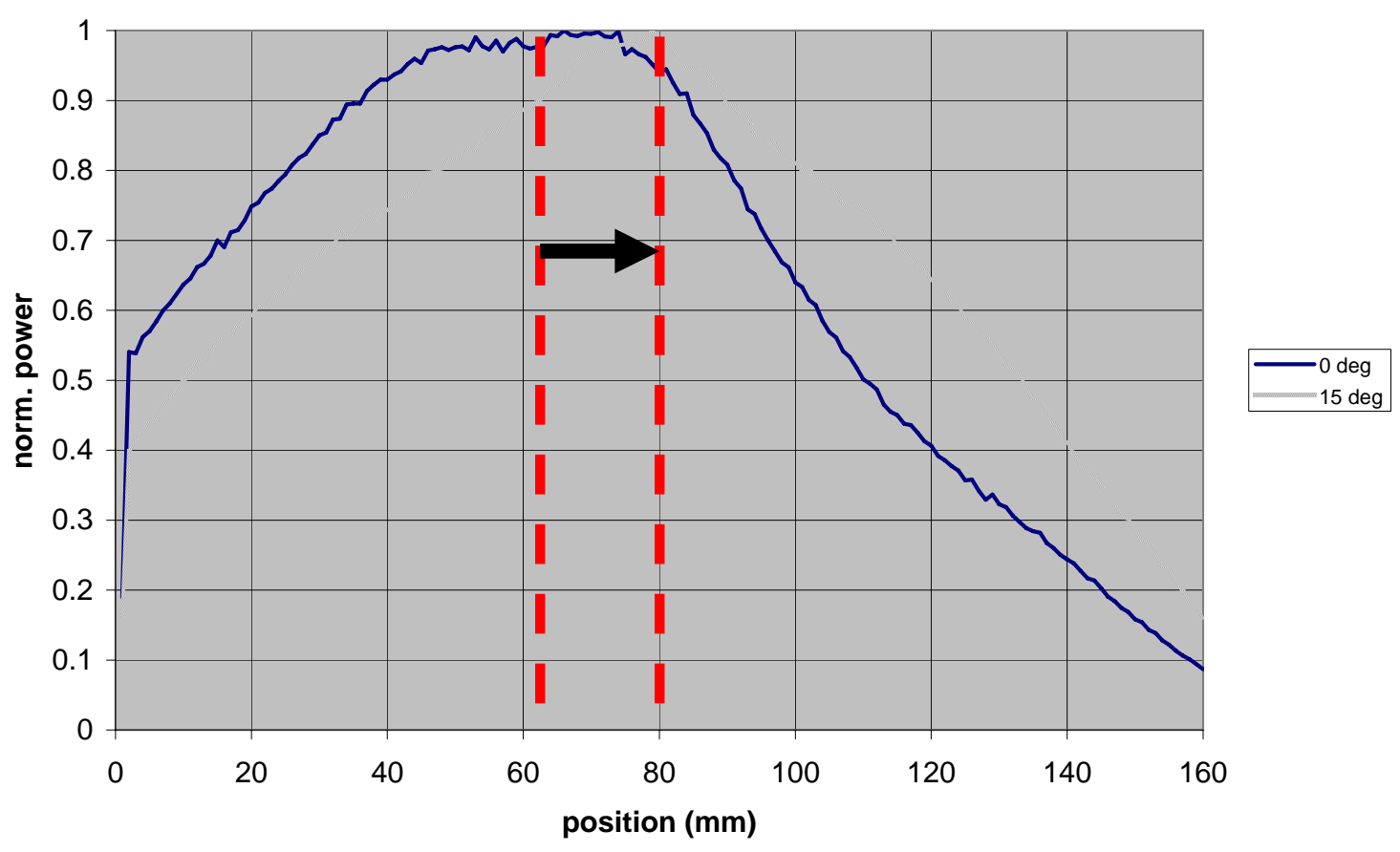

Fig.12. Typical normal incidence and 15 degree scans

index vs. frequency

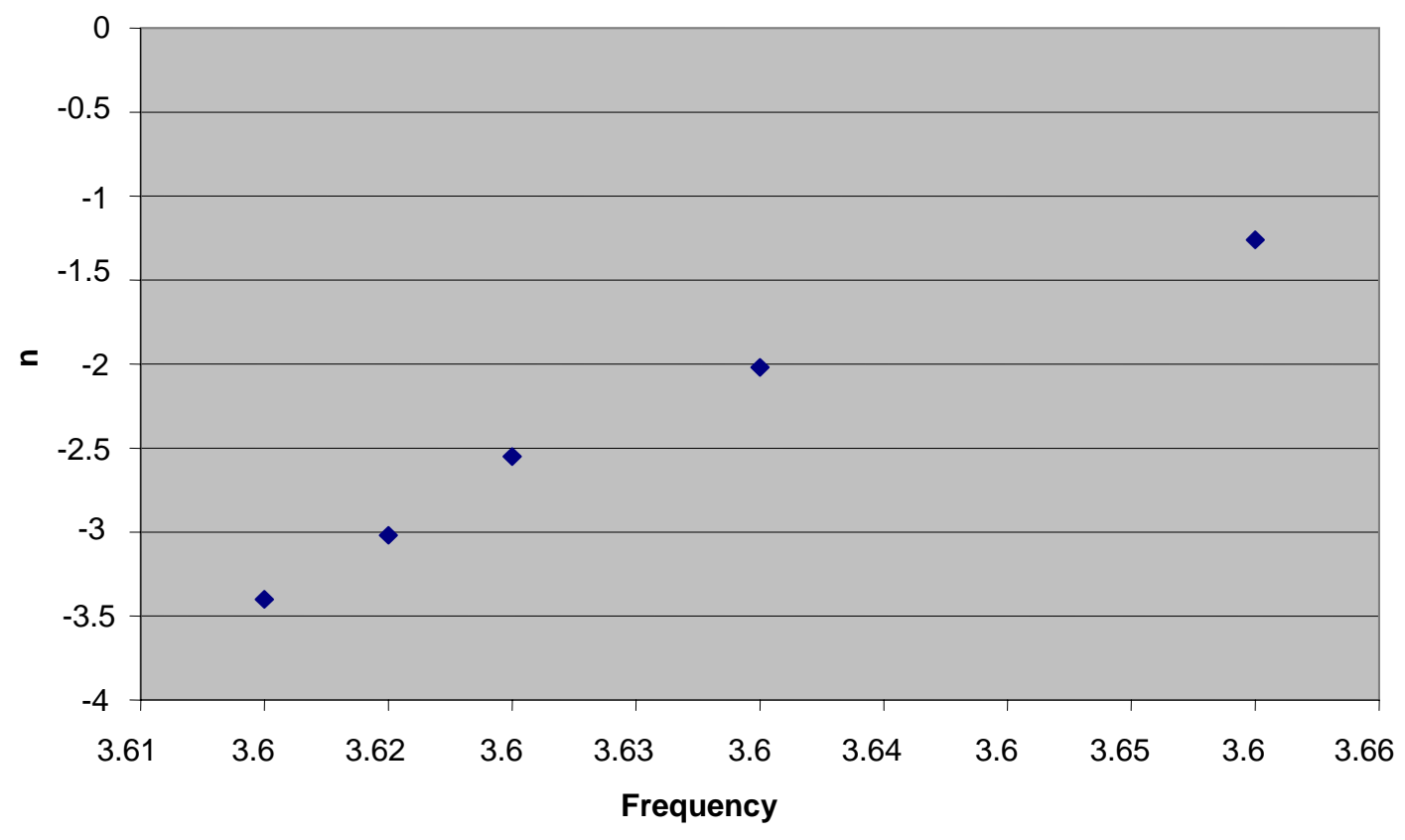

Fig.13 A plot showing the frequency dependence of the index of refraction 
One notices from the plot in Fig.12 that the energy density maximum shifts to the right when the horn antenna is also shifted to the right which is indicative of the negative index of refraction (the monopole is scanned from top to bottom as indicated in Fig.6 (a) and zero on the axis is taken to be the first point where the signal is acquired). From the plot in Fig. 13 one can see that the frequency which has the best transmission of energy through the lens ( $\mathrm{f}=3.625 \mathrm{GHz}$ ) is not necessarily the one that gives the index of refraction that is closest to $\mathrm{n}=-1(\mathrm{f}=3.66 \mathrm{GHz})$. Therefore, in all subsequent lens characterization experiments, a frequency of $3.66 \mathrm{GHz}$ was used to yield an $\mathrm{n}$ close to -1 .

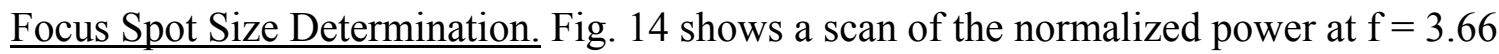
$\mathrm{GHz}$ at two distances away from the lens. The focus spot size was determined to be the peak width at half the maximum power, or the $-3 \mathrm{~dB}$ point.

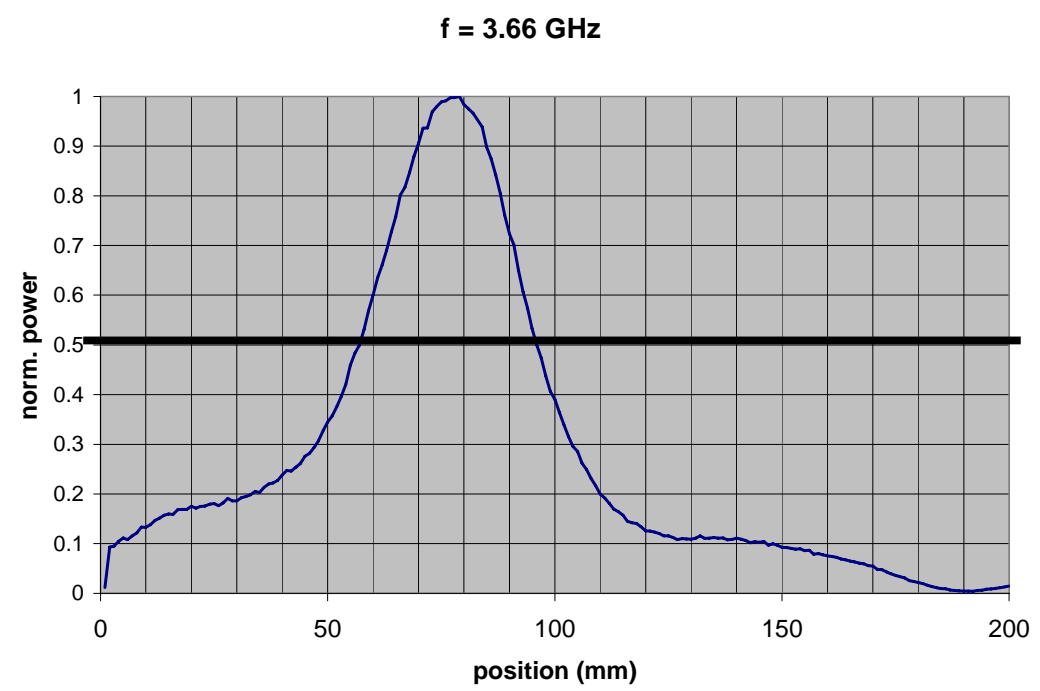

(a) 


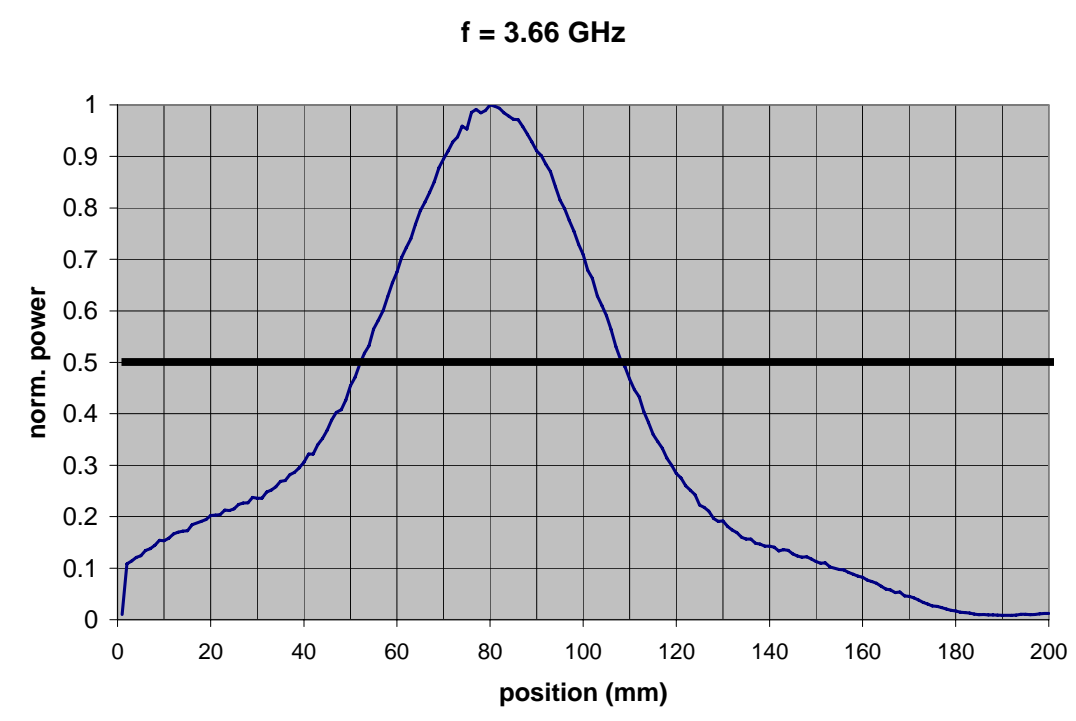

(b)

Fig.14 (a) Scan $12 \mathrm{~mm}$ away from the lens. (b) scan $32 \mathrm{~mm}$ away from the lens. Dark solid line depicts the $-3 \mathrm{~dB}$ point

The best focusing spot size of $0.48 \lambda$ was achieved for the frequency of $3.66 \mathrm{GHz}$ at a distance of $12 \mathrm{~mm}$ away from the lens. A better focusing spot size could possibly be achieved closer to the lens but this could not be investigated due to mechanical limitations of the experimental setup.

In order to emphasize the focusing abilities of the lens, scans at two different frequencies are compared (Fig.15). At $6 \mathrm{GHz}$, the metamaterial has positive $\varepsilon$ and $\mu$ and, therefore the energy transmitted through the material is unfocussed and relatively unattenuated.
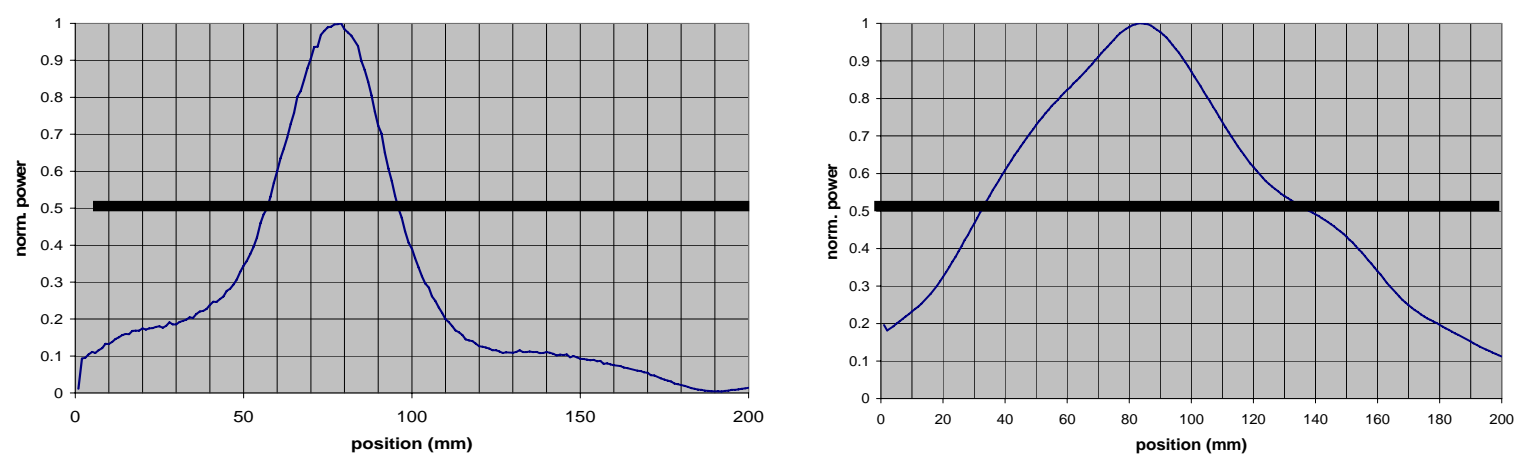
Fig.15 (a) Scan $12 \mathrm{~mm}$ away from the lens for $\mathrm{f}=3.66 \mathrm{GHz}$. (b) scan $12 \mathrm{~mm}$ away from the lens for $f=6 \mathrm{GHz}$. Dark solid line depicts $-3 \mathrm{~dB}$ point

From Fig. 15 one notices that at the $-3 \mathrm{~dB}$ point, the spot size for $\mathrm{f}=6 \mathrm{GHz}$ is $13.6 \mathrm{~cm}$ (which corresponds to $2.7 \lambda$ for $\lambda=5 \mathrm{~cm}$ ) whereas the spot size for $\mathrm{f}=3.66 \mathrm{GHz}$ is $3.9 \mathrm{~cm}$ (which corresponds to $0.48 \lambda$ for $\lambda=8.2 \mathrm{~cm}$ ). Fig. 16 shows the focused spot size versus distance from the lens for two frequencies $(f=3.64 \mathrm{GHz}$ and $\mathrm{f}=3.66 \mathrm{GHz})$

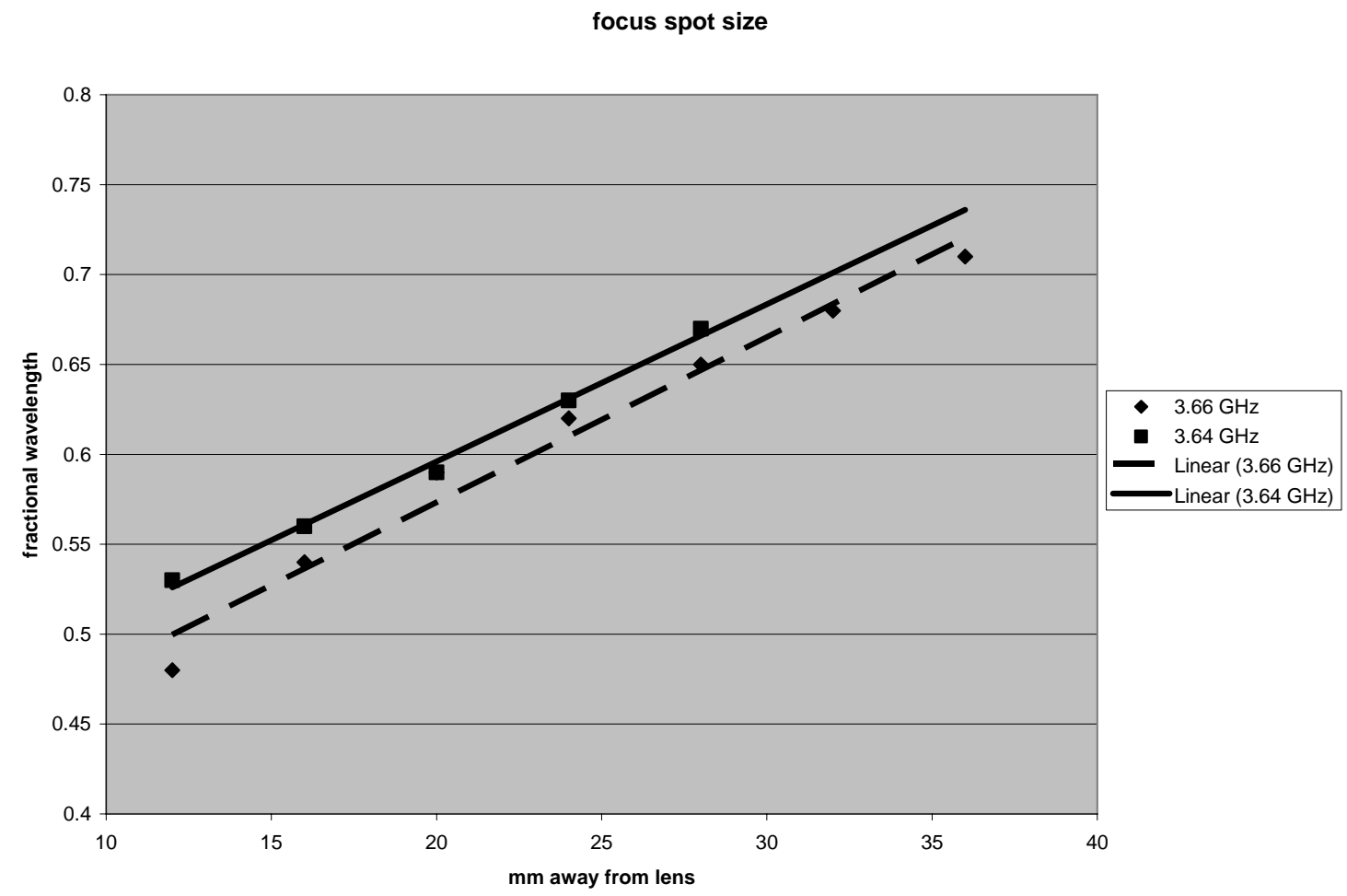

Fig.16 Focused spot size (in $\lambda$ ) vs. distance from the lens. The lines represent linear fitting of the data 
The fiberglass sample investigation. As mentioned in the Experimental Details section, the frequency of operation was changed slightly from the lens characterization experiments to $3.65 \mathrm{GHz}$. Though the frequency shift has the effect of moving $\mathrm{n}$ slightly further from the ideal $n=-1$ point, a small improvement in energy transmission was obtained. Fig.17 shows the scan of the fiberglass sample with the hole, after the background was subtracted.

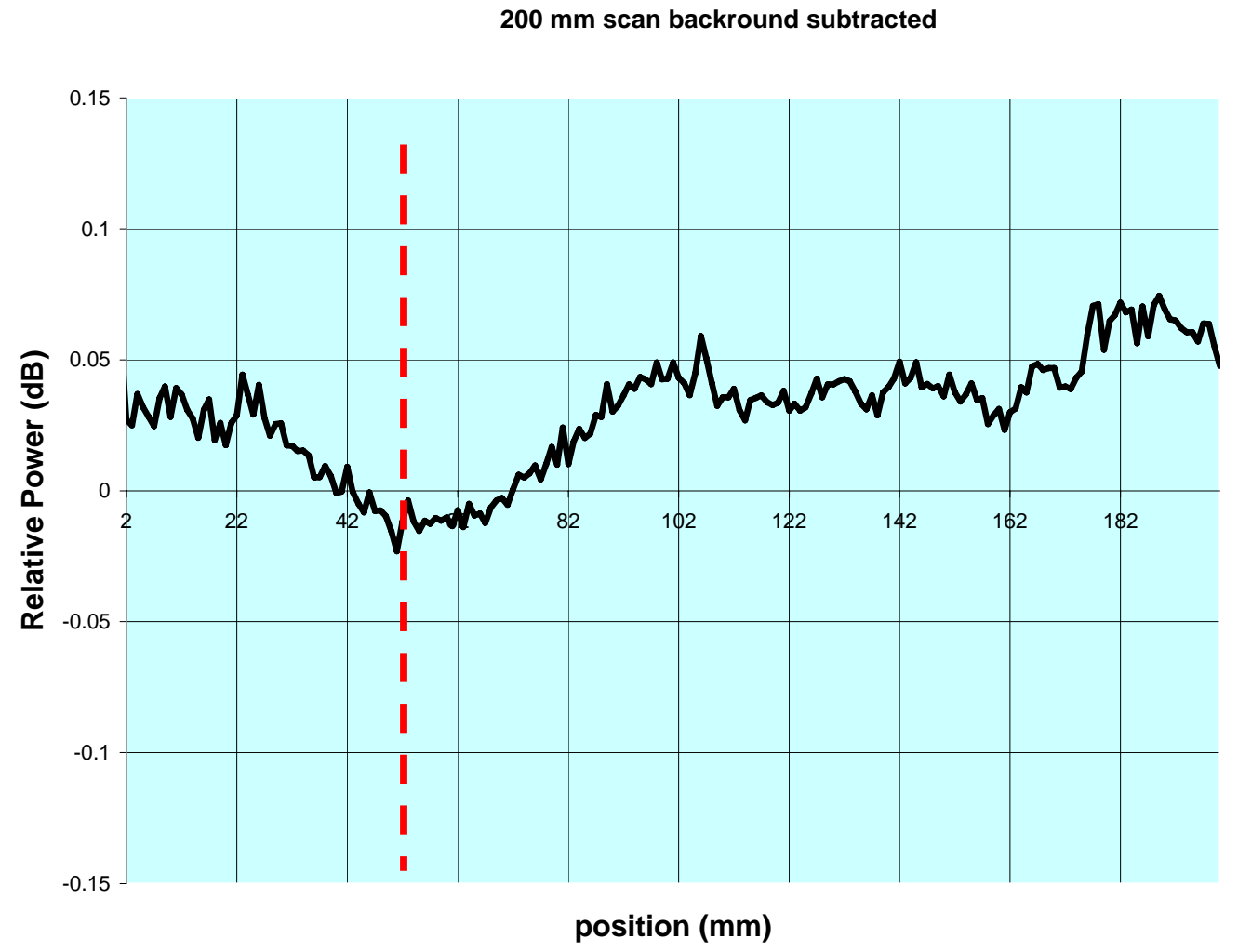

Fig.17 Relative Power scan of the sample at $f=3.65 \mathrm{GHz}$

From Fig. 17 one can determine the location of the hole from the minimum of the plot. Note that for this setup, the location of the receiving monopole was offset $3 \mathrm{~cm}$ from the peak transmission point of the lens. The procedure was repeated at a frequency of 6 $\mathrm{GHz}$, for which the system operates in a regular far field mode (the distance between the 
transmitting monopole and the sample was $15.8 \mathrm{~cm}$ ) to determine the presence of the defect. The result of the scan is shown in Fig. 18.

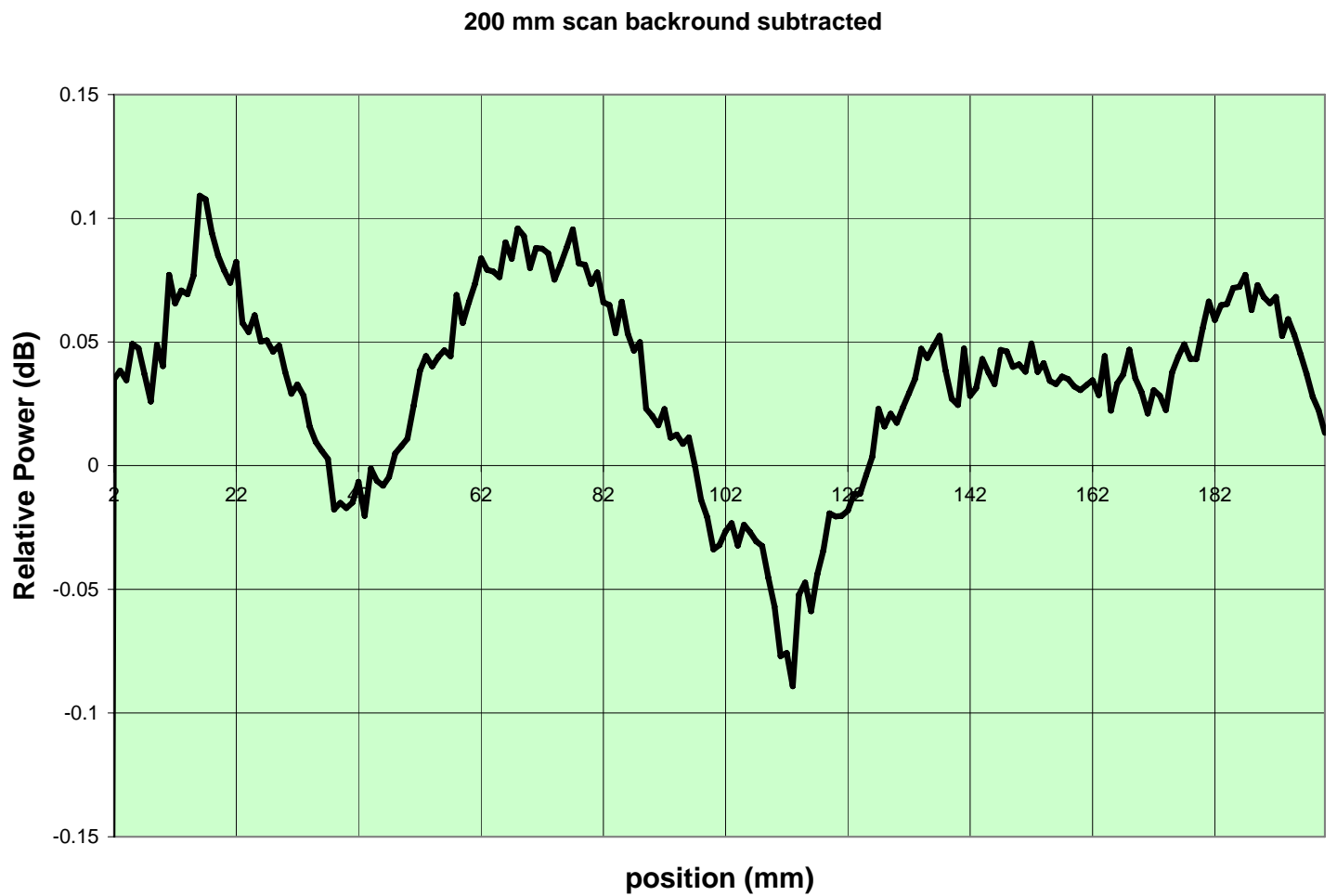

Fig.18 Relative Power scan of a sample as depicted in Fig.8 $\mathrm{f}=6 \mathrm{GHz}$

One notices that it is not possible to determine the location of the hole from the scan that is presented in Fig.18, which appears to be dominated by edge effects and/or by the structure of the lens that at this frequency acts like a grating.

In order to investigate the capabilities of this method further, a second hole of the same diameter was drilled $3 \mathrm{~cm}$ away from the first hole. The sample was scanned when both holes were filled in order to establish the background. Then the sample was scanned with one hole closed and one hole open and the second time with the other hole open and the first hole closed. The procedure was repeated when the frequency of operation was 
changed from $\mathrm{f}=3.65 \mathrm{GHz}$ to $\mathrm{f}=6 \mathrm{GHz}$. The results of the experiment are shown in Fig.19 and Fig.20.

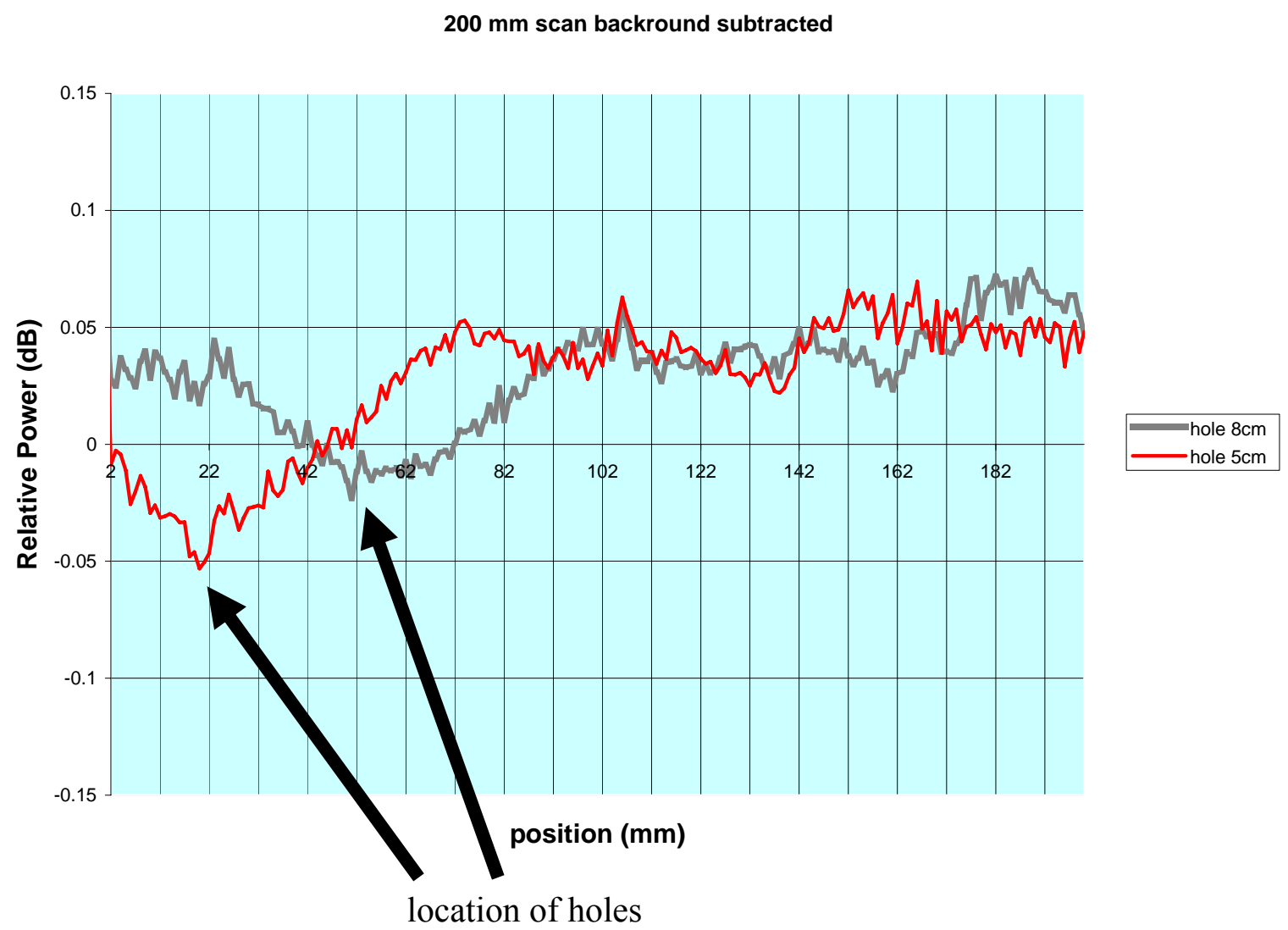

Fig.19 Relative Power scan of a sample when two holes were filled alternately ( $f=$ 3.65 GHz) 


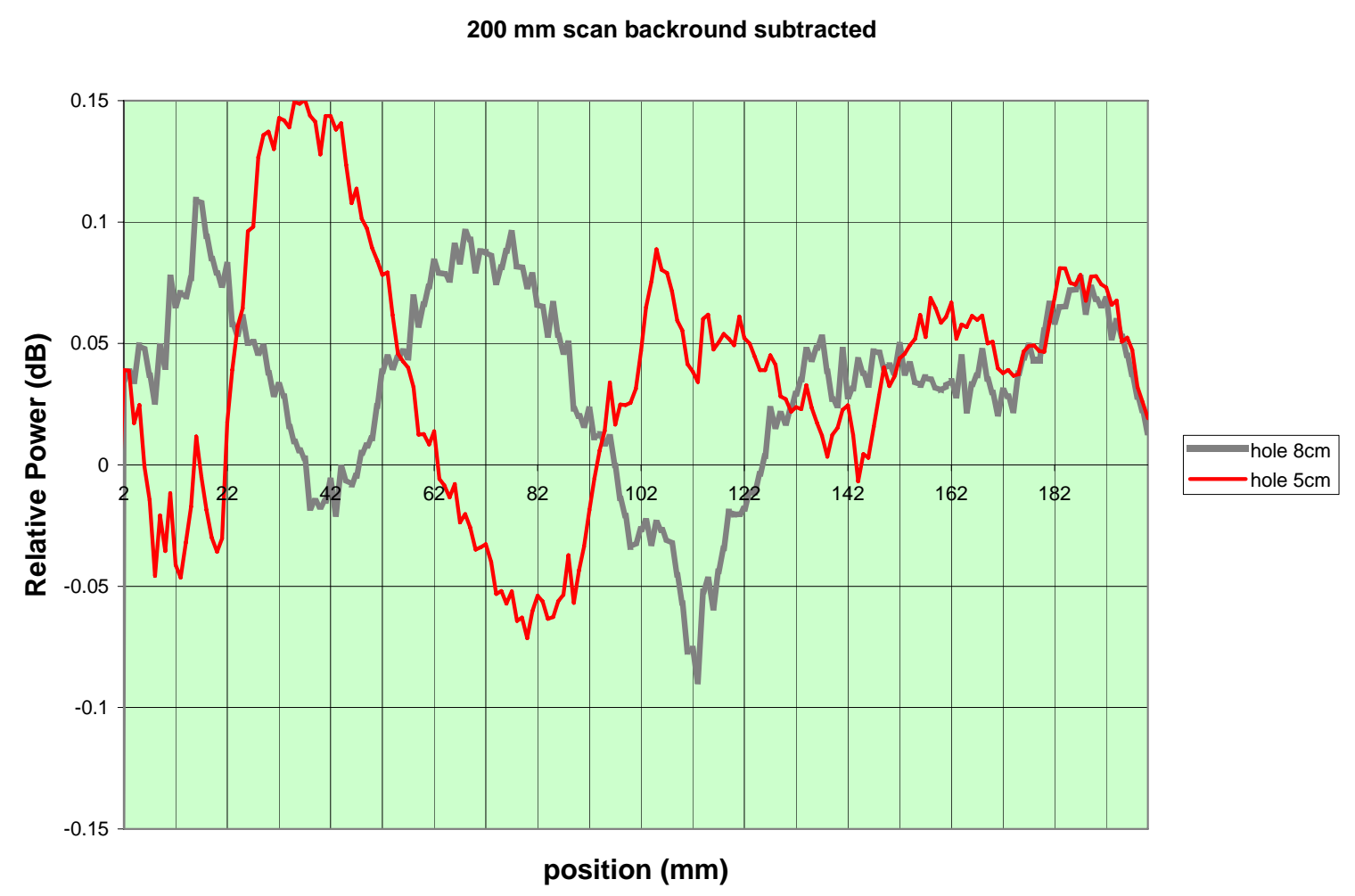

Fig.20 Relative Power scan of a sample when two holes were filled alternately ( $f=6$

\section{GHz)}

Fig. 20 shows that although a change in the hole's location causes some significant changes in the energy density, it is still very hard to verify the existence of the hole in the sample at $6 \mathrm{GHz}$. Part of this difficulty comes from the edge effects that remain even after the background is subtracted. At the same time one can identify the location of the hole when the sample is tested at $\mathrm{f}=3.65 \mathrm{GHz}$, a resonant frequency for this material. Discussion. The purpose of this work was a proof of concept for the principle of operation for a novel microwave NDE detector that is based on a metamaterial lens. It was successfully demonstrated that an EM wave emitted by a monopole $(\mathrm{f}=3.65 \mathrm{GHz})$ could be focused by a metamaterial lens with a sub-wavelength focus spot $(0.48 \lambda)$. This 
lens eliminates problems observed with far field methods (such as edge effects). On the other hand, this method is not sensitive to changes in standoff distance compared to results reported using conventional microwave methods. A hole with a diameter of $3 \mathrm{~mm}$ was detected using a wavelength of $8.2 \mathrm{~cm}$. However, with $\mathrm{f}=6 \mathrm{GHz}$, we could not obtain a similar result. The fact that one can detect defects much smaller than the wavelength of operation is not new and has been established previously in microwave near field $\mathrm{NDE}^{12}$. In this work, however, we have demonstrated sensitivity for a subwavelength defect at distances that are closer to far field mode.

One distinct disadvantage of this technique is the lossy nature of the metamaterial. The current effort is concerned with reducing these losses. This would allow the system to be further simplified by removing the receiving monopole (on the sample side of the lens) and using only one monopole for both transmitting and receiving the signal. A stronger signal to noise ratio would also enhance the sensitivity of the method.

It was shown in this paper that Ansoft's HFSS ${ }^{\mathrm{TM}}$ proves to be a good design tool for metamaterial lenses for the required frequencies of operation. The HFSS ${ }^{\mathrm{TM}}$ model will be used in the future to design a lens for a higher frequency of operation in order to enhance the sensitivity of the method. Another area for future investigation is to characterize the effects of the lens on the polarization of the incident radiation. Polarization shifts are suspected to be the cause for at least some of the "losses" which are experienced, in which case a more robust measurement system may need to include a more sophisticated receiving antenna. It may also be possible to exploit the polarization rotation which occurs upon transmission through the lens for enhanced penetration into some types of materials. 
In conclusion, for the first time the use of a metamaterial lens for NDE applications has been successfully demonstrated.

The authors would like to thank Terry Mack, Kenneth Dudley and Bob Young of the NASA Langley Research Center for their help in the design of the experimental setup and valuable advice through the duration of the experiments. We thank NASA Langley Research Center for their support for this work.

\section{References.}

1. M. Meyer and L. Herbeck, SAMPE Europe Conference and Exhibition.

2. E.C. Greenawald, L.J. Levenberry, N. Quaddoumi, A. McHardy, R. Zoughi and C.F. Poranski Jr., Review of Progress in Quantitative Nondestructive Evaluation, edited by D.O. Thompson and D.E. Chimenti, (2000) 1263.

3. R. Zoughi, J. Lai and K. Munoz, Materials Evaluation, February (2002) 171.

4. V.G. Veselago, Sov. Phys. Uspekhi, 10 (1968) 509.

5. J.B. Pendry, Physical Review Letters, 8517 (2000) 3966.

6. S. Ananta Ramakrishna, Rep. Prog. Phys. 68 (2005) 449.

7. J.B. Pendry, A.J.Holden, D.J. Robins, and W.J. Stewart, IEEE Trans. On Microwave Theory and Techniques, 47, 11 (1999) 2075.

8. J.B. Pendry, A.J.Holden, W.J. Stewart, I. Youngs, Physical Review Letters, 76, 25 (1996) 4773.

9. R.A. Shelby, D.R. Smith and S. Schultz, Science, 292 (2001) 77.

10. K. Aydin, I. Bulu and E. Ozbay, Optics Express, 13, 22 (2005) 8753. 
11. D. R. Smith, S. Schultz, P. Markos and C. M. Soukoulis, Physical Review B, 65 (2002) 195104.

12. M. Tabib-Azar, Review of Progress in Quantitative Non Destructive Evaluation, 20, edited by D.O. Thompson and D.E. Chimenti. 